\title{
Теоретическое описание трех основных механизмов удерживания в обращенно-фазовой ВЭЖХ на примере молекул нафталина, урацила и фенола
}

\author{
Пономарев Ф.В., Долгоносов А.М. \\ Институт геохимии и аналитической химии им. В.И. Вернадского Российской академии наук, Москва \\ Поступила в редакцию 18.07.2018 г.
}

DOI: https://doi.org/10.17308/sorpchrom.2018.18/591

В данной статье предложен новый подход к описанию удерживания аналитов в ВЭЖХ с учетом процессов адсорбции и сольватации. Для молекулы аналита рассматриваются межмолекулярные взаимодействия, включающие в себя неполярные Ван-дер-Ваальсовы взаимодействия, ориентационные и индукционные силы и водородную связь. При рассмотрении адсорбционных эффектов можно выделить три основных случая: удерживание неполярных молекул; удерживание сольватов, препятствующих адсорбции, и удерживание гетерополярных молекул. Результаты расчетов хорошо коррелируют с экспериментальными данными.

Ключевые слова: обращенно-фазовая ВЭЖХ, математическое моделирование адсорбции и сольватации, энергия межмолекулярных взаимодействий, хроматографическое удерживание.

\section{Theoretical description of three major retention mechanisms in reversed-phase HPLC on the example of naphthalene, uracil and phenol molecules}

\author{
Ponomarev P.V., Dolgonosov A.M. \\ V.I. Vernadsky Institute of Geochemistry and Analytical Chemistry, RAS, Moscow
}

Nowadays in the high performance liquid chromatography (HPLC) method there is a number of theoretical models allowing to predict chromatographic characteristics. For the last decade scientists have developed new models and have improved earlier existing approaches for this analytical method. However in all these models there is a one common aspect - a high degree of dependence on experimental data and a small possibility of parameters spreading on a wide range of systems.

It is convenient to describe the interaction of molecules at the level of quantum mechanical concepts of the chemical bond. The major approach is that the intermolecular interaction energy is defined by the dispersion, induction and orientation forces, and hydrogen bonding, which define character of all types of interaction between atoms in molecules and molecular complexes.

Intermolecular interaction energy correlates exponentially with distribution coefficient where the difference between the special way calculated adsorption energy of an analyte and a modifier has been taken, and even a small inaccuracy in energy definition considerably affects its value. The retention factor is connected with distribution coefficients over the value of column porosity.

In this article the new approach to the description of chromatographic retention of three types of analytes molecules for the HPLC isocratic elution mode is considered. In the adsorption effects examination it is possible to determine three main cases: non-polar molecule retention, retention of solvates hindering the adsorption and heteropolar retention of molecules. Non-empirical calculations have been carried out on the basis of various interactions contribution to the chemical bond with the usage of expressions from the theory of the generalized charges. For the molecules differing on hydrophilicity/hydrophobicity chromatographic 
characteristics have been finally defined. High degree of correlation between theoretical and experimental data has been found. It has been shown that the developed approach is relevant for application in different systems.

Keywords: reversed-phase HPLC, mathematical modeling of adsorption and solvation, the intermolecular interactions energy, chromatographic retention

\section{Введение}

В настоящее время в литературе, посвященной математическому моделированию высокоэффективной жидкостной хроматографии (ВЭЖХ) описываются теоретические модели, использующие большое количество эмпирических параметров [1-3]. В этих моделях существует один общий недостаток - высокая зависимость от экспериментальных данных и малая возможность переносимости параметров на широкий ряд систем.

Взаимодействие молекул определяет как термодинамические, так и кинетические параметры системы, которые удобно описывать на уровне квантовомеханических представлений о химической связи, поэтому в основе теоретических подходов лежит важнейшее представление об энергии межмолекулярного взаимодействия $(\mathrm{MMB})\left(E_{t o t}\right)$ как о совокупности дисперсионных, индукционных и ориентационных взаимодействий, которые наряду с водородными связями определяют характер всех типов связей между атомами в молекулах и молекулярных комплексах.

$$
E_{t o t}=E_{n p}+n_{H} E_{H}+E_{p},
$$

где $E_{n p}$ - энергия неполярных ван-дер-ваальсовых взаимодействий, $n_{H}$ - вероятность образования водородной связи, $E_{H}-$ энергия водородной связи, $E_{p}=E_{\text {ind }}+E_{o r}-$ энергия полярных взаимодействий, складывающаяся из энергии индукционных $E_{\text {ind }}$ и ориентационных $E_{\text {or }}$ взаимодействий.

Энергия ММВ экспоненциально коррелирует с коэффициентом распределения $\ln \Gamma \sim \Delta E_{\text {tot }}[4,5]$, где берется разность между нормированными специальным образом энергиями адсорбции аналита и модификатора, и даже небольшая неточность в определении энергии заметно сказывается на его величине. Фактор удерживания связан с коэффицентом распределения через величину порозности колонки.

$$
k=\frac{\Gamma}{\varepsilon},
$$

где $\varepsilon$ - порозность колонки, значение обычно находится в диапазоне 0.27-0.33.

Время удерживания $t_{R}$ линейно связано с фактором удерживания $k$ аналита [6]:

$$
t_{R}=t_{0}(k+1),
$$

где $t_{0}$ - мертвое время колонки.

Поскольку, в конечном итоге, соответствие теоретических выражений с экспериментальными параметрами проверяется по коэффициенту распределения аналита (или его времени удерживания), проблема корректного вычисления энергии адсорбции является крайне актуальной.

Существующие подходы к описанию удерживания в ВЭЖХ. Из ряда классических теорий можно выделить подходы Снайдера и Сочевинского $[7,8]$, которые рассматривают термодинамику конкурентной сорбции без подробного описания механизма распределения и пренебрегают эффектами физической адсорбции на сво- 
бодной поверхности; а также подход Скотта-Кучеры [9], который описывает конкретные модели адсорбции и сольватации.

Основное уравнение модели Снайдера:

$$
\ln k=a_{1}+a_{2} x+a_{3} x^{2},
$$

где $k$ - фактор удерживания, $a_{1}, a_{2}, a_{3}$ - эмпирические константы, $x$ - объемная доля модификатора.

Основное уравнение модели Скотта-Кучеры:

$$
\frac{1}{k}=A+B x,
$$

где $A, B-$ эмпирические константы.

Абрахам с коллегами показали, что в ходе моделирования системы может быть найден член, зависящий от энергии сольватации. Для описания хроматографических систем используется модель соотношений линейной сольватации (LSER), применяемая как для описания гидрофобных, так и гидрофильных взаимодействий. Данная модель изначально создавалась для газовой хроматографии, а при переходе к описанию жидкостной хроматографии может учитывать природу, качественный и количественный состав подвижной фазы [10-12].

Подход, основанный на теории обобщенных зарядов. Теория обобщенных зарядов [13-17] позволяет провести неэмпирический расчет энергии неполярных Вандер-Ваальсовых взаимодействий. По определению, обобщенные заряды - это характеристики молекул, которым пропорциональна энергия ММВ:

$$
E_{12}(r)=Q_{1} Q_{2} u_{b}(r),
$$

где $Q_{1}$ и $Q_{2}$ - обобщенные заряды молекул 1 и 2.

В формулу (6) входит $u_{b}(r)$ - функция межмолекулярного расстояния:

$$
u_{b}(r)=\frac{e^{2}}{a_{0}}\left[1.938 \cdot 10^{5} \cdot\left(\frac{r}{a_{0}}\right)^{-12}-5.115 \cdot\left(\frac{r}{a_{0}}\right)^{-6}\right],
$$

где $e-$ - заряд электрона, $a_{0}$ - радиус Бора (запись в системе СГСЭ).

Обобщенный заряд жесткого молекулярного фрагмента $(Q)$ может быть выражен через обобщенные заряды составляющих его атомов $\left(Q_{i}\right)$ в виде:

$$
Q^{\frac{4}{3}}=\sum_{i} Q_{i}^{\frac{4}{3}}
$$

Если молекула представляет собой набор жестких фрагментов, связанных нежесткими связями, то ее обобщенный заряд равен сумме обобщенных зарядов жестких фрагментов.

В свою очередь обобщенный заряд выражается через так называемый, электронный объем - аддитивную характеристику числа валентных электронов в жестком фрагменте молекулы.

$$
\begin{aligned}
& Q=V^{3 / 4}, \\
& V=\sum_{i} V_{i},
\end{aligned}
$$

где $V$ - электронный объем жесткого фрагмента; $V_{i}$ - электронный объем атома.

Электронный объем рассчитывается по формуле:

$$
V=N_{\sigma}+N_{\pi} \sqrt{2}
$$

где $N_{\sigma}$ - число б-электронов; $N_{\pi}$ - число $\pi$-электронов. В электронном объеме не учитываются те валентные электроны, расстояние которых до точки взаимодействия превышает радиус экранирования $r_{s}$.

Пономарев и др. / Сорбционные и хроматографические процессы. 2018. Т. 18. № 5 
В общем случае учета мезомерии для симметричных жестких фрагментов обобщенный заряд $Q$ рассчитывается по следующей формуле [13-17].

$$
Q=\left(V-\pi_{d} \sqrt{2}\right)^{\frac{3}{4}}+\left(\pi_{d} \sqrt{2}\right)^{\frac{3}{4}}=\left(N_{\sigma}+N_{\pi} \sqrt{2}-\pi_{d} \sqrt{2}\right)^{\frac{3}{4}}+\left(\pi_{d} \sqrt{2}\right)^{\frac{3}{4}},
$$

где $\pi_{d}$ - половина числа мезомерных электронов.

Целью данной работы является теоретическое описание поведения аналитов различной полярности в ВЭЖХ-системе с учетом процессов адсорбции на неполярном адсорбенте и сольватации в водно-органической подвижной фазе, использующее небольшое число физически значимых непереносимых эмпирических параметров.

\section{Теоретическая часть}

Модель ОФ ВЭЖХ для неполярных аналитов. Данная модель была описана в работах $[15,18,19]$ Напомним вкратце основные положения модели. Для обращенно-фазовой хроматографии (ОФ ВЭЖХ) основной вклад в адсорбцию молекул аналита или модификатора вносит неполярное взаимодействие.

В модели рассматривается конкурентная адсорбция между молекулами аналита и модификатора. Показатель стехиометрии обмена $p$ является отношением молекулярных площадок рассматриваемых молекул. Согласно теории обобщенных зарядов обобщенному заряду пропорциональна молекулярная площадка $\left(\mathrm{Hм}^{2}\right)$ :

$$
w=0.0307 Q .
$$

Отсюда следует выражение для $p$ (индекс М означает принадлежность к молекуле аналита, индекс L — к молекуле модификатора).

$$
p=\frac{Q_{M}}{Q_{L}} .
$$

С помощью величины молекулярной площадки можно определить отношение емкости монослоя $a$ к концентрации $c_{1}$ чистого модификатора:

$$
\frac{a}{c_{1}}=\frac{s M_{L}}{10^{-18} \cdot 0.0307 N_{A} Q_{L} \rho},
$$

где $s$ - площадь поверхности силикагеля, $\mathrm{m}^{2} / \mathrm{cm}^{3} ; M_{L}$ - молярная масса молекулы модификатора, г/моль; $N_{A}$ - число Авогадро, моль ${ }^{-1} ; Q_{L}-$ обощенный заряд моди-

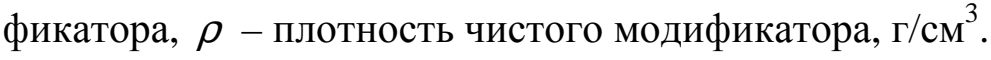

Относительное удерживание аналита $y\left(y=\frac{\Gamma}{\Gamma_{0}}\right.$ где $\Gamma$ - реальный коэффициент распределения аналита и $\Gamma_{0}$ - коэффициент распределения в системе с чистым растворителем без модификатора) определяется по формуле:

$$
\ln y=-p \ln \left[1+x\left(\kappa_{L}-1\right)\right]+\theta\left[x \ln \alpha_{M}-\kappa_{M}{ }^{-1}(1-x)\left(1-\alpha_{M}\right)\right],
$$

где $\theta=\frac{\kappa_{L} x}{1+\left(\kappa_{L}-1\right) x}-$ доля занятой поверхности в сорбенте (предполагается изотерма Ленгмюра); $x$ - объемная доля модификатора в подвижной фазе; $\alpha_{M}-$ конкурентное отношение аналита к модификатору, которое можно найти по формуле:

$$
\ln \alpha_{M}=G_{L}(p-1)+\frac{1}{2} \ln \frac{M_{M}}{p M_{L}},
$$

$G_{L}=-0.537$ при $300 \mathrm{~K}$ - константа модификатора рассчитываемая с использованием системной константы, определяемой из условий для растворителя ( $D=-0.33$ - для 
воды); нормированная константа Генри к, связанная с обобщенным зарядом, является сорбционным параметром, который показывает взаимосвязь между теоретическими и экспериментальными характеристиками:

$$
\kappa=\exp (B Q+D)
$$

где $B=-\frac{U_{0, A}}{R T}-\frac{g_{M}}{2}-$ энергетический фактор молекулы в поле адсорбента, где $U_{0, A}=-1.67$ кДж/моль - потенциал поверхности (эмпирический параметр для линейных углеводородных фаз); $g_{M}=\left(\sum \frac{m_{i}}{M} V_{i}\right)^{-\frac{3}{4}}-$ фактор теплоемкости молекулы, где $m_{i}$ - масса атома, $M$ - масса молекулы.

Коэффициент распределения аналита в системе при нулевом содержании модификатора $\Gamma_{0}$, определяется по формуле:

$$
\Gamma_{0}=\kappa \frac{a}{c_{1}} .
$$

При расчете адсорбции учитывали только неполярные взаимодействия, пренебрегая очень слабыми индукционными взаимодействиями, однако для расчета эффектов сольватации учитывались кроме неполярных взаимодействий вклады неполярных и гидрофильных взаимодействий.

Описание межмолекулярных взаимодействий с учетом полярности и гидрофильности. Энергия ММВ, которая реализуется в явлении сольватации рассчитывается по формуле (система СИ):

$$
\begin{aligned}
& E_{\text {tot }}=\left[u_{b m} Q_{L}\left(Q_{M} f(r)+2 \frac{c_{1}}{\lambda^{3}} \mu_{M}^{2}\right)+\frac{a N_{A}}{4 \pi \varepsilon_{0} r_{b}^{6} \lambda^{3}} \mu_{L}^{2}\left(Q_{M}+\frac{c_{2} \mu_{M}^{2}}{4 \pi \varepsilon_{0} \lambda^{3} k_{B} T}\right)\right] \cdot\left(\frac{r_{b}}{r}\right)^{6}+ \\
& +\frac{3 n_{H} N_{A}}{2 c_{3} \pi \varepsilon_{0}}\left(\frac{r_{b}}{r}\right)^{2}
\end{aligned}
$$

где слагаемые в квадратных скобках характеризуют дисперсионный, ориентационный и индукционный вклады в энергию; последний член характеризует вклады водородных связей; индексы А и В относятся к различным молекулам; $\lambda$ - отношение расстояний между центрами диполей и центрами обобщенных зарядов молекул (величина, обычно близкая к единице); $k_{B}$ - константа Больцмана; $\varepsilon_{0}-$ диэлектрическая константа; $r$ - переменная величина - расстояние между молекулярными объектами; $f(r)$ - отношение потенциала типа Леннард-Джонса, полученного в теории обобщенных зарядов к дисперсионному члену этого выражения [20-22]:

$$
f(r)=\frac{u_{b}(r)}{u_{b m}}=2-\left(\frac{r}{r_{b}}\right)^{-6},
$$

где $u_{b m}=-88.54$ Дж/моль - теоретическая константа.

Коэффициенты, использованные в (20), выражаются через фундаментальные постоянные:

$$
c_{1}=\frac{0.9676}{e^{2} a_{0}^{2}} ; \quad c_{2}=\frac{0.1347}{a_{0}^{3}} ; \quad c_{3}=\frac{1354 a_{0}}{e^{2}} ; \quad c_{T}=\frac{c_{2}}{k_{B} T} ; \quad a=4.948 a_{0}^{3}
$$

Первая производная величины (20) по расстоянию, приравниваемая к нулю, необходима для поиска равновесного расстояния $r$ :

$$
n_{H} z^{10}+A B z^{6}-B=0
$$


где $z=\frac{r}{r_{b}}, r_{b}=0.3442$ нм.

Коэффициенты для данного уравнения равны соответственно:

$$
\begin{gathered}
A=1+c_{1}\left(v_{1}+v_{2}+c_{T} v_{1} v_{2}\right), \\
B=-c_{3} u_{0} Q,
\end{gathered}
$$

где $v_{1}$ и $v_{2}$ - характеристики полярности, которые определяются через дипольный момент молекулы:

$$
v=\frac{\mu^{2}}{Q} .
$$

Общая энергия адсорбции $\left(E_{a d s}\right)$ с учетом вклада подвижной фазы $\left(E_{M-m p}\right)$ и неподвижной фазы $\left(E_{M-s p}\right)$ определяется, как приведено ниже.

$$
E_{a d s}=E_{M-s p}-E_{M-m p} .
$$

Взаимодействие аналита с подвижной фазой (ПФ) включает в себя вклады модификатора $E_{L}$ и растворителя $E_{W}$.

$$
E_{M-m p}=p_{L} E_{L}+p_{W} E_{W} .
$$

В ОФ ВЭЖХ обычно в качестве растворителя используется вода. Отношение чисел компонентов подвижной фазы в сольватной оболочке Р может быть найдено с учетом их больцмановских факторов.

$$
P=\left(\frac{x}{1-x}\right) \cdot \exp \left[\frac{-\left(E_{L}-E_{W}\right)}{R T}\right] .
$$

Весовые коэффициенты участия во взаимодействии ПФ с аналитом модификатора $p_{L}$ и воды $p_{W}$ определяются как решение системы уравнений:

$$
\left\{\begin{array}{l}
\frac{p_{L}}{p_{W}}=P \\
p_{L}+p_{W}=1
\end{array} .\right.
$$

В системе без модификатора $(x=0)$ с учетом полярных и гидрофильных взаимодействий выражение для коэффициента распределения вместо (19) запишется следующим образом:

$$
\Gamma_{0}=\frac{a}{c_{1}} \exp \left(\frac{-E_{a d s}}{R T}-\frac{g_{M} Q}{2}+D\right)
$$

В дальнейшем рассчитываются коэффициенты распределения и время удерживания, как описано выше в (2)-(3) и через относительное удерживание аналита (16).

Учет сольватационных эффектов. В зависимости от того, является аналит гомополярной молекулой или гетерополярной (молекула, у которой существуют разнесенные в пространстве полярная и неполярная части), возможно два варианта адсорбции молекул на неполярной неподвижной фазе из полярной среды. Гомополярная молекула может образовывать или не образовывать сольватные комплексы. Последний случай характерен для неполярных гидрофобных молекул и был рассмотрен в [15].

В случае с образованием сольватных комплексов молекулы ПФ, входящие в состав сольвата, в зависимости от расположения могут влиять на адсорбцию аналита. На рис.1 (I) схематично показан комплекс, в котором молекулы ПФ расположены в плоскости молекулы аналита и не препятствуют ее адсорбции. Если молекулы ПФ, входящие в комплекс, расположены между аналитом и адсорбентом (рис.1 (II)), то 
они должны быть вытеснены в процессе адсорбции аналита. Результирующий эффект определяется разностью энергии адсорбции аналита и энергии взаимодействия с ним удаляемых молекул ПФ. Присоединение таких молекул ПФ происходит с вычитанием их дипольных моментов из дипольного момента аналита. Общее число присоединяемых («сверху» и «снизу») молекул не превышает отношения данных величин: $\left[\frac{\left|\mu_{M}\right|}{\left|\mu_{L}\right|}\right]=k \geq 1$, так как процесс присоединения прекращается при полной компенсации дипольного момента центральной молекулы. Энергия комплекса молекулы ПФ и аналита включает в себя вклад ориентационных, индукционных взаимодействий и водородных связей (20).

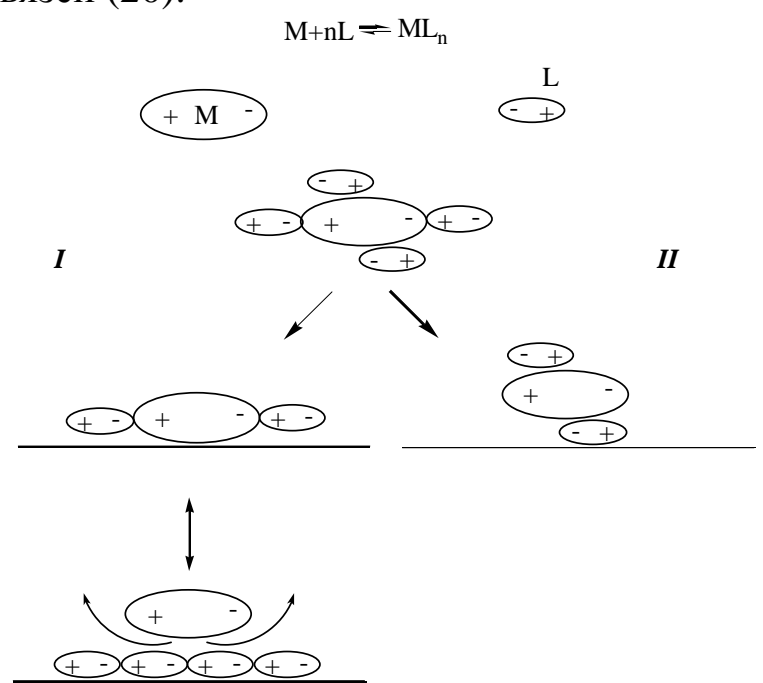

Рис. 1. Структура сольватных комплексов аналита М с молекулой жидкой фазы L

В качестве примера можно привести образование комплекса урацила с водой. Модель сольвата содержит много молекул воды, которые могут находиться между молекулой адсорбата и поверхностью адсорбента и препятствовать процессу адсорбции на сорбенте. Однако согласно схеме, представленной на рис.1(II) необходимо учитывать только ту воду, которая может помешать адсорбции. В работе [23] показано, что молекулы воды с увеличением их числа могут располагаться перпендикулярно плоскости пиримидинового кольца урацила.

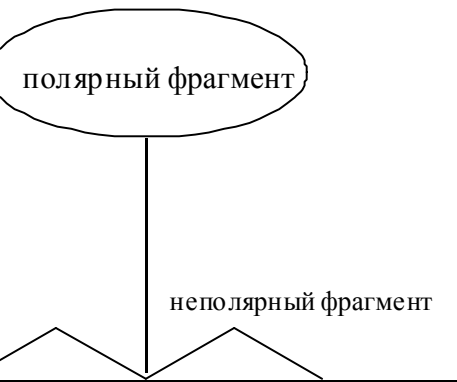

Рис. 2. Адсорбция гетерополярных молекул на неполярном сорбенте

Для гетерополярных молекул происходит поляризация в поле адсорбента так, что ось, связывающая неполярные и полярные фрагменты в диаметрально противоположных частях молекулы, стремится расположиться перпендикулярно поверхности адсорбента (рис.2). Этот эффект связан с различным сродством полярных и неполярных фрагментов молекулы к полярным и неполярным частям системы, соот- 
ветственно. При таком расположении молекулы ее обобщенный заряд может уменьшиться за счет попадания электронов, удаленных от поверхности адсорбента, в зону экранирования. В таком случае энергия взаимодействия будет скорректирована при учете данного фактора в значении величины обобщенного заряда.

\section{Обсуждение результатов}

Все расчеты проводились при температуре эксперимента $T=308.15$ К. Для учета полярных взаимодействий в системах с водно-ацетонитрильным элюентом (80 об.\% ацетонитрила) был проведен расчет теоретического фактора удерживания и теоретических времен удерживания для аналитов нафталина, урацила и фенола [24]. Хроматограмма эксперимента представлена на рисунке 3.

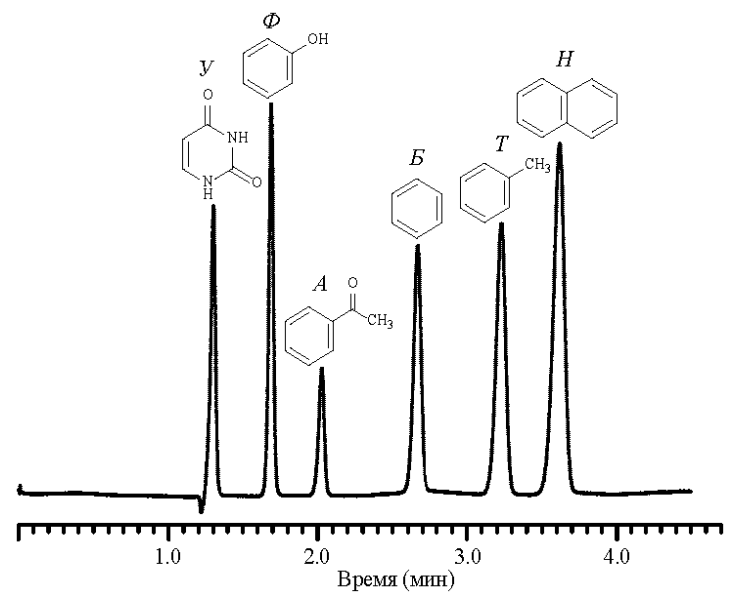

Рис. 3. Изучаемая экспериментальная хроматограмма, где

У - урацил, Ф - фенол, А - ацетофенон, Б - бензол, Т - толуол, Н - нафталин

Величины дипольных моментов молекул были взяты из справочных данных $[25,26]$. Дипольный момент неподвижной фазы C18 равен нулю $\mu_{H \Phi}=0$, также данный сорбент не способен к образованию с аналитом водородной связи.

Нафталин<smiles>c1ccc2ccccc2c1</smiles>

Адсорбция нафталина характеризуется неполярными взаимодействиями. Методология расчета впервые была дана в [22], использована для оптимизации состава многокомпонентного элюента в [27], а также кратко приведена в теоретической части данной статьи.

В таблице 1 приведена последовательность расчета хроматографического удерживания аналита в системе ОФ ВЭЖХ: сначала рассчитываются обобщенные заряды аналита и модификатора по формулам (9-11), затем определяется показатель стехиометрии обмена по формуле (14). Для ацетонитрила рассчитывается отношение $\frac{a}{c_{1}}$ по (15) и нормированная на метанол константа Генри ацетонитрила $\kappa_{\mathrm{CH}_{3} \mathrm{CN}}$ по (18), конкурентное отношение нафталина к ацетонитрилу по (17) и вспомогательные параметры $g$ и $B$, учитывающие свойства адсорбента. В результате подстановки всех найденных величин в уравнение (16) для $\ln y$ находят относительное удерживание нафталина $y$. После чего определяются хроматографические параметры по формулам (2-3), (19). 
Таблица 1. Результаты расчета хроматографических характеристик для нафталина

\begin{tabular}{|c|c|c|c|}
\hline $\begin{array}{c}\text { Расчет обобщенных зарядов (9-11), } \\
\text { (14) }\end{array}$ & $\begin{array}{c}\text { Вычисление па- } \\
\text { раметров конку- } \\
\text { рентной сорбции } \\
(15-18)\end{array}$ & $\begin{array}{c}\text { Результаты } \\
\text { расчета хро- } \\
\text { матогра- } \\
\text { фических па- } \\
\text { раметров } \\
(2-3),(19)\end{array}$ & $\begin{array}{c}\text { Данные экспе- } \\
\text { римента и от- } \\
\text { носительная } \\
\text { ошибка расче- } \\
\text { та }\end{array}$ \\
\hline $\begin{array}{l}N_{\sigma C_{10} H_{8}}=38 ; N_{\pi C_{10} H_{8}}=10 ; \pi_{d C_{10} H_{8}}=5 \\
Q_{C_{10} H_{8}}=21.73 \\
N_{\sigma C H_{3} C N}=10 ; N_{\pi C H_{3} C N}=4 ; \pi_{d C_{3} C N}=0 \\
Q_{C_{3} C N}=7.87 \\
p_{C_{10} H_{8} / C H_{3} C N}=2.76\end{array}$ & $\begin{array}{l}\frac{a}{c_{1}}=0.08 \\
g=0.34 \\
\alpha_{C_{10} H_{8} / C H_{3} C N}=2.11 \\
B=0.48 \\
\ln y=-8.10 \\
\kappa_{C H_{3} C N}=29.05\end{array}$ & $\begin{array}{c}\Gamma_{0}=2046 \\
\Gamma=0.62 \\
k_{\text {meоp }}=1.88 \\
t_{\text {meор }}=3.71 \mathrm{мин}\end{array}$ & $\begin{array}{c}t_{\text {ээсп }}=3.60 \mathrm{МИН} \\
\Delta_{r}=3.1 \%\end{array}$ \\
\hline
\end{tabular}

Описанная процедура расчета может быть применена для любых гомополярных неполярных молекул.

Урацил $\stackrel{\mathrm{N}}{\mathrm{H}}$. Представителем гомополярных полярных молекул является урацил. При описании адсорбции молекулы урацила необходимо учитывать эффекты, связанные с дипольными моментами и водородными связями.

Молекула урацила имеет следующие характеристики $N_{\sigma}=24 ; N_{\pi}=6 ; \pi_{d}=3$; $Q=13.61 ; \mu_{U r}=4.16$ Д. Сначала определяется взаимодействие несольватированной молекулы урацила с фазой $\mathrm{C}_{18}$. В результате решения уравнения (22) определяется величина $z=0.97$, которая соответствует равновесному расстоянию $r=0.334$ нм и энергии $E_{a d s 0}=32.3$ кДж/моль.

В соответствии с теоретическим описанием вариантов влияния сольватной оболочки на адсорбцию рассмотрим комплексы урацила с водой, в которых молекулы воды препятствуют подходу урацила к поверхности адсорбента. При образовании таких комплексов результирующий дипольный момент равен разности моментов объединяющихся молекул. Рассчитаем возможные энергии таких комплексов, учитывая лишь те из них, которые образуются без перемены знака результирующей величины дипольного момента.

$$
\begin{gathered}
\mathrm{Ur}+\mathrm{H}_{2} \mathrm{O} \rightarrow \mathrm{Ur} \cdot \mathrm{H}_{2} \mathrm{O} \\
\mathrm{Ur} \cdot \mathrm{H}_{2} \mathrm{O}+\mathrm{H}_{2} \mathrm{O} \rightarrow \mathrm{Ur} \cdot 2 \mathrm{H}_{2} \mathrm{O} \\
\mathrm{Ur}+\mathrm{CH}_{3} \mathrm{CN} \rightarrow \mathrm{Ur} \cdot \mathrm{CH}_{3} \mathrm{CN}
\end{gathered}
$$

Результаты расчета термодинамических параметров адсорбции представлены в таблице 2. Поскольку величина $E_{U r \cdot \mathrm{CH}_{3} C N}$ мала по сравнению с энергией комплекса $E_{U r \cdot H_{2} O}$, то она, как показывают вычисления, вносит лишь малый вклад в общую величину энергии.

Перед определением общей энергии системы рассчитаем весовые коэффициенты, учитывающие вклад в систему комплексов урацила с водой и ацетонитрилом в элюирующей смеси по (28)-(29).

$$
P=0.02
$$

Пономарев и др. / Сорбционные и хроматографические процессы. 2018. Т. 18. № 5 
Таблица 2. Расчет термодинамических параметров системы для взаимодействия урацила с молекулой воды и ацетонитрила

\begin{tabular}{|c|c|c|}
\hline \multirow{2}{*}{ Параметр } & \multicolumn{2}{|c|}{ Значение } \\
\hline & Вода & Ацетонитрил \\
\hline $\begin{array}{c}\text { Дипольный момент } \\
\text { молекулы, Д }\end{array}$ & 1.85 & 3.93 \\
\hline $\begin{array}{c}\text { Дипольный момент комплекса } \\
\mathrm{Ur} \cdot \mathrm{L}\end{array}$ & 2.31 & 0.23 \\
\hline $\begin{array}{c}\text { Дипольный момент комплекса } \\
\mathrm{Ur} \cdot 2 \mathrm{~L} \\
\end{array}$ & 0.45 & - \\
\hline Значение аргумента z по (25) & 0.95 & 0.94 \\
\hline Искомое расстояние $\mathrm{r}$ по (25), нм & 0.327 & 0.324 \\
\hline $\begin{array}{c}\text { Численное значение стандартной } \\
\text { функции расстояния для выраже- } \\
\text { ния энергии межмолекулярных } \\
\text { взаимодействий, } f\end{array}$ & 0.64 & 0.55 \\
\hline $\begin{array}{l}\text { Энергия образования комплекса } \\
\mathrm{Ur} \cdot \mathrm{H}_{2} \mathrm{O} \cdot E_{U r \cdot H_{2} O} \text { по }(24), \text { Дж/моль }\end{array}$ & 56.1 & - \\
\hline $\begin{array}{c}\text { Энергия присоединения молеку- } \\
\text { лы воды к комплексу Ur· } \mathrm{H}_{2} \mathrm{O} \\
E_{U r \cdot \mathrm{H}_{2} O} \text { по (24), Дж/моль }\end{array}$ & 25.2 & - \\
\hline $\begin{array}{c}\text { Дипольный момент межмолеку- } \\
\text { лярного комплекса } \mathrm{Ur} \cdot \mathrm{CH}_{3} \mathrm{CN} \\
\mu_{U r \cdot \mathrm{CH}_{3} \mathrm{CN}}, \text { Д }\end{array}$ & - & 0.23 \\
\hline $\begin{array}{c}\text { Энергия образования комплекса } \\
\mathrm{Ur} \cdot \mathrm{CH}_{3} \mathrm{CN} E_{U r \cdot \mathrm{CH}_{3} \mathrm{CN}} \text { по (24), } \\
\text { кДж/моль }\end{array}$ & - & 1.19 \\
\hline
\end{tabular}

Для нахождения искомых коэффициентов решим систему уравнений:

$$
\begin{gathered}
\left\{\begin{array}{l}
\frac{p_{\mathrm{CH}_{3} \mathrm{CN}}}{p_{\mathrm{H}_{2} \mathrm{O}}}=0.02 \\
p_{\mathrm{H}_{2} \mathrm{O}}+p_{\mathrm{CH}_{3} \mathrm{CN}}=1
\end{array}\right. \\
p_{\mathrm{H}_{2} \mathrm{O}}=0.98 ; p_{\mathrm{CH}_{3} \mathrm{CN}}=0.02
\end{gathered}
$$

Отсюда следует, что вода вытесняет ацетонитрил из сольватной оболочки. Подстановка найденных значений в формулу (27) дает энергию сольвата. Энергия адсорбции с учетом удаления молекулы воды из пространства между урацилом и адсорбентом $E_{a d s}=7.34$ кДж/моль. Расчетные данные в системе равны $\Gamma_{0}=0.01 ; \Gamma=3.77 \cdot 10^{-5} ; k_{\text {meоp }}=1.14 \cdot 10^{-4} ; t_{R_{\text {meop }}}=1.30$ мин. Экспериментальные данные составляют $t_{R}$ эксn $=1.29$. Относительная ошибка результатов расчета $\Delta_{r}=0.8 \%$ менее $5 \%$ свидетельствует о приемлемости данной модели.

Фенол<smiles>Oc1ccccc1</smiles>

. Молекулы, результаты неэмпирического расчета которых описаны выше, имеют равномерно распределенные полярные и неполярные участки, поэтому подходят для рассмотрения поляризации в неполярном адсорбенте относительно полярной жидкости. Фенол - это молекула с характерной гидрофильной ОН группой на конце, следовательно, она имеет ярко выраженные полярные и непо- 
лярные участки. То есть данная молекула расположена так, что неполярный фрагмент расположен ближе к адсорбенту, а полярный - ближе к жидкости.

В таком случае важно правильно рассчитать электронный объем молекулы $V$ с учетом экранированных электронов. Оценив размеры молекулы фенола и величину радиуса экранирования $r_{S} \sim \frac{1}{\sqrt{E_{\kappa}}} \sim 0.3 \div 0.4 \mathrm{HM} \mathrm{в} \mathrm{соответствии} \mathrm{с} \mathrm{размерами} \mathrm{молекулы}$ фенола, показанными на рисунке 4, электроны на максимально удаленной группе -ОН экранированы, и они не учитываются в электронном объеме.

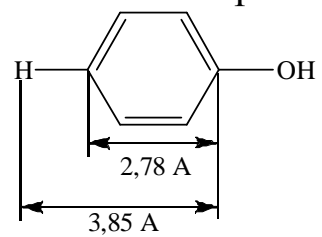

Рис. 4. Расстояния между атомами в фенильном фрагменте молекулы фенола

Для молекулы фенола исходные данные и результаты расчета приведены в таблице 3.

Таблица 3. Результаты расчета хроматографических характеристик для фенола

\begin{tabular}{|c|c|c|c|}
\hline $\begin{array}{c}\text { Расчет обобщенных за- } \\
\text { рядов }(9-11),(14)\end{array}$ & $\begin{array}{c}\text { Вычисление па- } \\
\text { раметров конку- } \\
\text { рентной сорбции } \\
(15-18)\end{array}$ & $\begin{array}{c}\text { Результаты рас- } \\
\text { чета хроматогра- } \\
\text { фических пара- } \\
\text { метров (2-3), } \\
\text { (19) }\end{array}$ & $\begin{array}{c}\text { Данные экспери- } \\
\text { мента и относи- } \\
\text { тельная ошибка } \\
\text { расчета }\end{array}$ \\
\hline $\begin{array}{l}N_{\sigma}=23 ; N_{\pi}=6 ; \pi_{d}=0 \\
\text { число экранированных } \\
\text { электронов } \Delta N_{\sigma}=3 \\
Q=13.30 \\
p=1.69\end{array}$ & $\begin{array}{l}\frac{a}{c_{1}}=0.11 \\
\alpha_{P h / C H_{3} C N}=1.52 \\
g=0.40 \\
B=5.66 \\
\ln y=-5.35 \\
\kappa_{C H_{C N} C N}=29.05\end{array}$ & $\begin{array}{c}\Gamma_{0}=31.43 \\
\Gamma=0.15 \\
k_{\text {meop }}=0.45 \\
t_{R_{\text {meоp }}}=1.87 \mathrm{MИН}\end{array}$ & $\begin{aligned} t_{R_{\text {эксп }}} & =1.70 \mathrm{МИН} \\
\Delta_{r} & =10.0 \%\end{aligned}$ \\
\hline
\end{tabular}

В молекуле фенола отсутствует ось симметрии, поэтому эффект мезомерии не проявляется. Величина относительной ошибки результатов расчета, равная $10.0 \%$, определяет возможность использования рассмотренного подхода к молекулам со структурными фрагментами разной полярности в рамках модели неэмпирического расчета хроматографических параметров аналитов в ОФЖХ.

\section{Заключение}

В работе были рассмотрены три типа молекул, поведение которых практически включает в себя все случаи, характерные для ОФ ВЭЖХ. Расчет неполярной системы произведен на примере с нафталином. При переходе к адсорбции полярных молекул необходимо рассматривать взаимодействие этих молекул не только с адсорбентом, но и с молекулами подвижной фазы. В случае водного ацетонитрильного элюента расчет урацила и фенола сводился к оценке структуры их сольватов и учету влияния на адсорбцию гидратной воды. Произведенные априорные вычисления удовлетворительно коррелируют с экспериментальными данными по удерживанию

Пономарев и др. / Сорбционные и хроматографические процессы. 2018. Т. 18. № 5 
рассмотренных молекул разной полярности в обращенно-фазовой высокоэффективной жидкостной хроматографии.

Работа выполнена при финансовой поддержке РФФИ (грант 18-03-00382a).

\section{Список литературы}

1. Close E.J., Salm J.R., Bracewell D.G., Sorensen E. // Chemical Engineering Research and Design. 2014. Vol. 92. No 7. pp. 13041314.

2.Nikitas P., Pappa-Louisi A. // J. Chromatogr. A. 2009. Vol. 1216. No 10. pp. 1737-1755.

3. Boswell P.G., Abate-Pella D., Hewitt J.T. // J. Chromatogr. A. 2015. Vol. 1412. № Supplement C. pp. 52-58.

4. Gritti F., Guiochon G. // Anal. Chem. 2006. Vol. 78. № 13. pp. 4642-4653.

5. Kloubek J. // J. Chromatographia. 1992. Vol. 33, № 9-10. pp. 478-484

6. Janczuk B., Choma I., Dawidowicz A.L., Kliszcz A et al. // Chromatographia. 1990. Vol. 30. № 7/8. pp. 382-387.

7. Snyder L.R. Principles of adsorption chromatography: The separation of nonionic organic compounds. Madison Avenue. New York: Marcel Dekker. Inc. 1968. 413 p.

8. Soczewinski E. // Anal. Chem. 1969. Vol. 41, № 1. pp. 179-182.

9. P. W. Scott R., Kucera P. // J. Chromatogr. A. 1975. Vol. 112. pp. 425-442.

10.Abraham M.H., Ibrahim A., Zissimos A.M. // J. Chromatogr. A. 2004. Vol. 1037. № 1. pp. 29-47.

11.D'Archivio A.A., Maggi M.A., Ruggieri F. // Analytica Chimica Acta. 2011. Vol. 690. № 1. pp. 35-46.

12.Schuster G., Lindner W. // J. Chromatogr. A. 2013. Vol. 1273. pp. 73-94.

13.Долгоносов А.М., Рудаков О.Б., Суровцев И.С., Прудковский А.Г. Колоночная аналитическая хроматография как объект математического моделирования. Воронеж. ГЕОХИ РАН. Воронежский ГАСУ. 2013. 400 c.

14.Долгоносов А.М. Модель электронного газа и теория обобщенных зарядов для описания межатомных взаимодействий и адсорбции. М. ЛИБРОКОМ. 2009. 167 с.

15.Долгоносов А.М. Неспецифическая селективность в проблеме моделирования вы- сокоэффективной хроматографии. М. КРАСАНД. 2012. $256 \mathrm{c.}$

16.Долгоносов А.М. // Сорбиионные и хроматографические прочессы. 2015. Vol. 15, № 2. pp. $168-178$.

17.Долгоносов А.М. // Журн. физ. химии. 2001. Т. 75, № 10. pp. 1813-1820

18. Долгоносов А.М. // Сорбиионные и хроматографические проиессы. 2011. Т. 11. № 4. C. 435-448.

19. Долгоносов А.М. // Сорбичонные и хроматографические проиессы. 2011. Т. 11. № 4. C. 449-458.

20.Долгоносов А.М., Зайцева Е.А. // Сорбиионные и хроматографические процессы. 2014. T. 14. № 4. pp. 578-590.

21. Долгоносов А.М., Зайцева Е.А. // Сорбиионные и хроматографические прочессы. 2015. Т 15. № 3. pp. 321-332.

22. Долгоносов А.М. // Сорбичинные и хроматографические проиессы. 2015. Vol. 15. № 3. pp. 312-320.

23.Тен Г.Н., Кадров Д.М., Баранов В.И. // Биофизика. 2014. Т. 59. № 4. pp. 656-665.

24.Henry R.A. Keys to High Speed HPLC with Isocratic Mobile Phases. Supelco, Division of Sigma-Aldrich, 595 North Harrison Road, Bellefonte, PA 16823: SIGMA-ALDRICH, 2010.

25. Haynes W.M. Dipole moments // Handbook of Chemistry and Physics. CRS Press; Taylor \& Francis Group, 2014. Vol. 95th Edition. pp. 9-51-9-59.

26. Kulakowska I., Geller M., Lesyng B., Wierzchowski K.L. // Biochimica et Biophysica Acta (BBA) - Nucleic Acids and Protein Synthesis. 1974. Vol. 361. № 2. pp. 119-130.

27.Пономарев Ф.В., Долгоносов А.М. // Сорбиионные и хроматографические проиессы. 2018. Т. 18. № 1. С. 15-25. 


\section{References}

1. Close E.J., Salm J.R., Bracewell D.G., Sorensen E., Chemical Engineering Research and Design, 2014, Vol. 92, No 7, pp. 1304-1314.

2. Nikitas P., Pappa-Louisi A., J. Chromatogr. A., 2009, Vol. 1216, No 10, pp. 17371755.

3. Boswell P.G., Abate-Pella D., Hewitt J.T., J. Chromatogr. A, 2015, Vol. 1412, No Supplement C, pp. 52-58.

4. Gritti F., Guiochon G., Anal. Chem., 2006, Vol. 78, No 13, pp. 4642-4653.

5. Kloubek J., J. Chromatographia, 1992, Vol. 33, No 9-10, pp. 478-484.

6. Janczuk B., Choma I., Dawidowicz A.L., Kliszcz A et al., Chromatographia, 1990, Vol. 30, No 7/8, pp. 382-387.

7. Snyder L.R. Principles of adsorption chromatography: The separation of nonionic organic compounds. Madison Avenue, New York, Marcel Dekker, Inc., 1968, 413 p.

8. Soczewinski E., Anal. Chem., 1969, Vol. 41, No 1, pp. 179-182.

9. Scott R P.W., Kucera P., J. Chromatogr. A, 1975, Vol. 112, pp. 425-442.

10. Abraham M.H., Ibrahim A., Zissimos A.M., J. Chromatogr. A., 2004, Vol. 1037, No 1, pp. 29-47.

11. D'Archivio A.A., Maggi M.A., Ruggieri F., Analytica Chimica Acta, 2011, Vol. 690, No 1, pp. 35-46.

12. Schuster G., Lindner W., J. Chromatogr. A, 2013, Vol. 1273, pp. 73-94.

13. Dolgonosov A.M., Rudakov O.B., Surovtsev I.S., Prudkovskii A.G. Kolonochnaya analiticheskaya khromatografiya kak ob"ekt matematicheskogo modelirovaniya. Voronezh: GEOKhI RAN - Voronezhskii GASU, 2013, $400 \mathrm{p}$.

14. Dolgonosov A.M. Model' elektronnogo gaza i teoriya obobshchennykh zaryadov dlya opisaniya mezhatomnykh vzaimodeistvii i adsorbtsii. M., LIBROKOM, 2009, 167 p.

Пономарев Филипп Валерьевич - аспирант, лаборатория сорбционных методов, ГЕОХИ РАН, Москва

Долгоносов Анатолий Михайлович д.Х.н., ведущий научный сотрудник, лаборатория сорбционных методов, ГЕОХИ РАН, профессор кафедры химии, Университет «Дубна», Москва
15. Dolgonosov A.M. Nespetsificheskaya selektivnost' $\mathrm{v}$ probleme modelirovaniya vysokoeffektivnoi khromatografii, M., KRASAND, 2012, $256 \mathrm{p}$.

16. Dolgonosov A.M., Sorbtsionnye i khromatograficheskie protsessy, 2015, Vol. 15, No 2, pp. 168-178.

17.Dolgonosov A.M., Rus.J.Phys.Chem., 2001, Vol. 75, No 10, pp.1659-1666.

18. Dolgonosov A.M., Sorbtsionnye i khromatograficheskie protsessy, 2011, Vol. 11, No 4, pp. 435-448.

19. Dolgonosov A.M., Sorbtsionnye i khromatograficheskie protsessy, 2011, Vol. 11, No 4, pp. 449-458.

20. Dolgonosov A.M., Zaitseva E.A., Sorbtsionnye i khromatograficheskie protsessy, 2014, Vol. 14, No 4, pp. 578-590.

21. Dolgonosov A.M., Zaitseva E.A., Sorbtsionnye i khromatograficheskie protsessy, 2015, Vol. 15, No 3, pp. 321-332.

22. Dolgonosov A.M., Sorbtsionnye i khromatograficheskie protsessy, 2015. Vol. 15, No 3, pp. 312-320.

23. Ten G.N., Kadrov D.M., Baranov V.I., Biofizika, 2014, Vol. 59, No 4, pp. 656-665.

24. Henry R.A. Keys to High Speed HPLC with Isocratic Mobile Phases. Supelco, Division of Sigma-Aldrich, 595 North Harrison Road, Bellefonte, PA 16823: SIGMA-ALDRICH, 2010.

25. Haynes W.M. Dipole moments // Handbook of Chemistry and Physics. CRS Press; Taylor \& Francis Group, 2014, Vol. 95th Edition, pp. 9-51-9-59.

26. Kulakowska I., Geller M., Lesyng B., Wierzchowski K.L., Biochimica et Biophysica Acta (BBA) - Nucleic Acids and Protein Synthesis, 1974, Vol. 361, № 2. pp. 119-130.

27. Ponomarev P.V., Dolgonosov A.M., Sorbtsionnye i khromatograficheskie protsessy, 2018, Vol. 18, No 1, pp. 15-25.

Ponomarev Phillip V. - postgraduate student, Lab of Sorption Methods, GEOKHI RAS, Moscow, e-mail: filpon@mail.ru

Dolgonosov Anatoly M. - Dr.Sci.(Chem.), Leading scientific associate, Lab of Sorption Methods, GEOKHI RAS, Prof. of Chemistry Chair, University «Dubna», Moscow, e-mail: amdolgo@mail.ru 